\title{
Quantitative Measurements of Magnetic Vortices USing Position RESOLVED DIFFRACTION IN LORENTZ STEM
}

\author{
NESTOR J. ZALUZEC
}

\section{Materials Science Div., Argonne National Lab, Argonne, IL, USA}

A number of electron column techniques have been developed over the last forty years to permit visualization of magnetic fields in specimens. These include: Fresnel imaging (Fuller and Hale ${ }^{1}$ ), Differential Phase Contrast $\left(\right.$ Chapman $\left.^{2}\right)$, Electron Holography (Tonomura ${ }^{3}$ ) and Lorentz STEM $\left(\right.$ Zaluzec $\left.^{4}\right)$. In this work we have extended the LSTEM methodology using Position Resolved Diffraction (PRD) to quantitatively measure the in-plane electromagnetic fields of thin film materials.

The experimental work reported herein has been carried out using the ANL AAEM HB603Z $300 \mathrm{kV}$ FEG instrument ${ }^{5}$. In this instrument, the electron optical column was operated in a zero field mode (figure 1), at the specimen, where the objective lens is turned off and the probe forming lens functions were reallocated to the $\mathrm{C} 1, \mathrm{C} 2$, and $\mathrm{C} 3$ lenses. Post specimen lenses (P1, P2, P3, P4) were used to magnify the transmitted electrons to a YAG screen, which was then optically transferred to a Hamamatsu ORCA ER CCD array. This CCD was interfaced to an EmiSpec Data Acquisition System and the data was subsequently transferred to an external computer system for detailed quantitative analysis. In Position Resolved Diffraction mode, we digitally step a focused electron probe across the region of interest of the specimen while at the same time recording the complete diffraction pattern at each point in the scan.

Figure 2 shows a Bright Field LSTEM image of the lithographically produced array of $\sim 100 \mathrm{~nm}$ thick Permalloy microdots on an $\sim 75 \mathrm{~nm}$ thick SiN amorphous film, supported on a 1000 mesh $\mathrm{Cu}$ grid. No magnetic contrast is shown in this image, but the polycrystalline nature of the microdots is clearly visible. Independent confirmation by Magnetic Force Microscopy indicated that the out of plane magnetic fields is minimal for the microdot array. Quantitative analysis of the in-plane electromagnetic field distribution is obtained by measuring the vector components of Lorentz deflection (amplitude and orientation) of the transmitted electron diffraction patterns (EDPs) at each of the 900 points of a two dimensional 30x30 pixel LSTEM scan of selected areas of the SiN film and a individual microdots. In order to perform these measurements correctly it was essential that the instrument be precisely aligned and that all instrumentally induced displacements of the EDP be negligible. This was confirmed by operating the instrument in PRD mode and simply translating the stage until an empty grid square was present, essentially measuring "vaccum". The results of such a null specimen measurement are shown in the polar plot of Figure 3a, where the total vector displacement can be discerned to be minimal (the group of 900 vectors is centered at the origin). In contrast, figure $3 \mathrm{~b}$ shows the measurement of the Lorentz displacement vector of the EDPs measured from an $\sim 0.5 \mu \mathrm{m}^{2}$ area of the $\mathrm{SiN}$ film (region A of fig. 2), while in figure 4c, from a neighboring microdot (region $B$ of fig. 2). In figure $3 \mathrm{~b}$, we see that the scattering is highly distorted and asymmetric due in part to charging of the SiN film, while in comparison, in $3 \mathrm{c}$, we see that the scattering is symmetric distributed equally in nearly all amplitudes and orientations.

To more succinctly present the data of figures $3 \mathrm{~b}$ and $3 \mathrm{c}$, we replot the analysis as two-dimensional pseudo-images of the "Lorentz" amplitude and orientation for the SiN film (4a,b) and microdot (4c,d) respectively. In the $\mathrm{SiN}$ film we see that both pseudo-images show little systematic correlation as is expected from the polar plot (3b). On the other hand for the microdot, we see that the amplitude increases from near zero at the microdot center (black) to a maximum (bright) at the periphery and decreasing thereafter. In addition and equally importantly we observe that the orientation dependence of the displacement vector for the microdot rotates continuously around the center of the field of measurement (4d). These last two pseudo-images $(4 \mathrm{c}, \mathrm{d})$ can therefore be interpreted to indicate that the microdot contains a simple magnetic vortex, the Lorentz field of which, is rotating continuously about the nominal center of the microdot. We also note, in passing, that the SiN film is charging and we can thus see that the PRD methodology is also applicable to measuring in-plane electrostatic fields. 


\section{References:}

1.) H. Fuller and M. Hale, J Applied Phys., 31 No. 10, 1699, (1960).

2.) J. Chapman, J. Phys. D. 17, 623 (1984)

3.) J. Tonomura, J. Magn. Mag. Mater. 35, 963 (1983),

4.) N.J. Zaluzec, Microscopy \& Microanalysis, 7, Number 5, Sup 1, (2001)

5.) N.J. Zaluzec, Proc. of $26^{\text {th }}$ MAS Conf. SF Press. 137, (1991)

6.) This work was supported in part by the U.S. DoE under BES-MS W-31-109-Eng-38 at ANL.

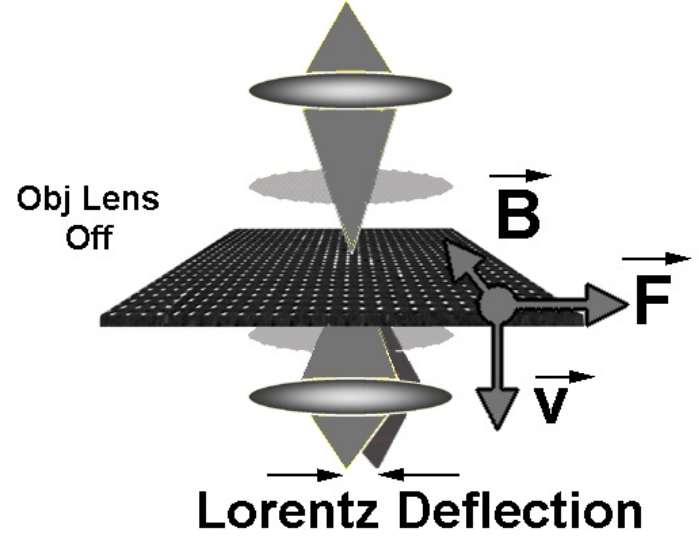

Figure 1. Electron Optical Configuration in Lorentz mode.

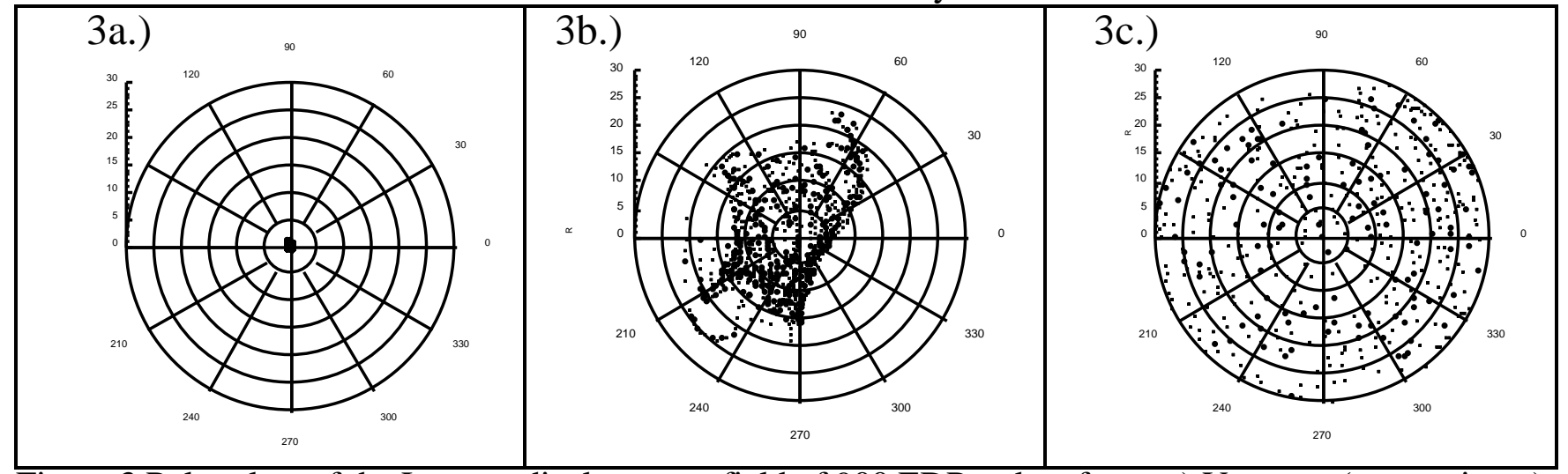

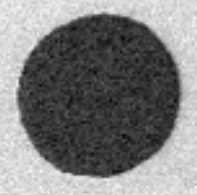

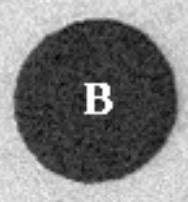

A

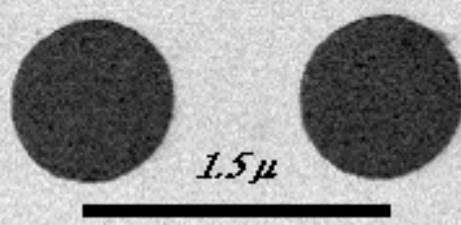

Figure 2. BF Lorentz STEM Image of Permalloy microdots on SiN film

Figure 3 Polar plots of the Lorentz displacement field of 900 EDPs taken from: a) Vaccuun (no specimen), b) Area A of the SiN film [fig. 2], and c) Microdot B [fig.2]. Each point in these plots represents a single measurement of an EDP, in 3a the 900 data points are too closely spaced to be resolved.

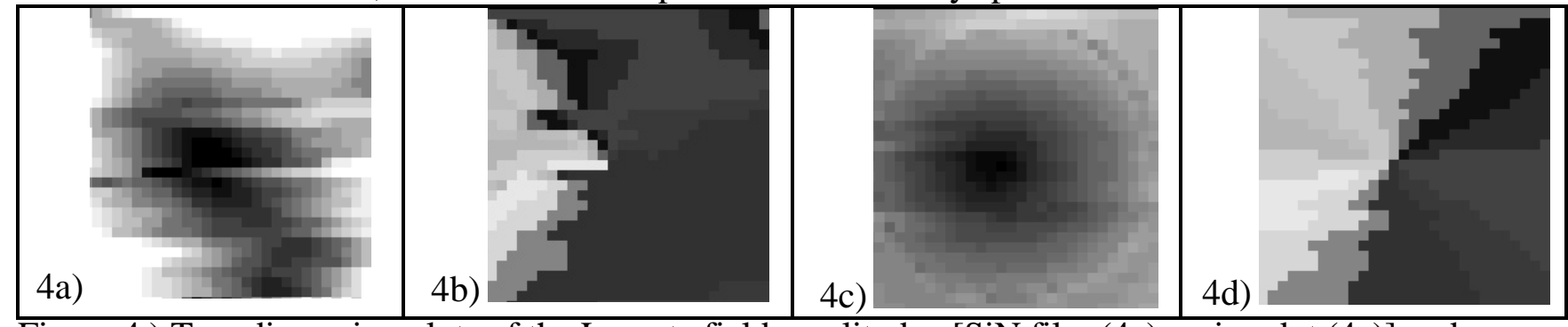

Figure 4.) Two dimension plots of the Lorentz field amplitude [SiN film (4a), microdot (4c)] and orientation [SiN film (4b), microdot (4d)]. All amplitudedata is directly grayscale coded and normalized from minimum (black) to maximum (white), while the rotation data is grayscale converted from a 360 degree full spectrum color wheel. For SiN film the orientation component is rotationally asymmetric and directional reflecting the data of fig $3 \mathrm{~b}$, while that of the microdot is clearly rotationally symmetric. Together, the pseudo-images of $4 \mathrm{c}, \mathrm{d}$ characterizes the Lorentz field to be an inplane vortex located near the center of the microdot. 